\title{
Report of a patient undergoing chronic responsive deep brain stimulation for Tourette syndrome: proof of concept
}

\author{
${ }^{*}$ Rene Molina, MS, ${ }^{1,2}$ Michael S. Okun, MD, ${ }^{2-4}$ Jonathan B. Shute, PhD, ${ }^{2,5}$ Enrico Opri, MS, ${ }^{2,5}$ \\ P. Justin Rossi, PhD, ${ }^{2}$ Daniel Martinez-Ramirez, MD, ${ }^{2,3}$ Kelly D. Foote, MD, ${ }^{2,4}$ and \\ Aysegul Gunduz, PhD'-3,5
}

Departments of ${ }^{1}$ Electrical and Computer Engineering, ${ }^{3}$ Neurology, and ${ }^{4}$ Neurosurgery; ${ }^{2}$ Center for Movement Disorders and Neurorestoration; and ${ }^{5}$ J. Crayton Pruitt Family Department of Biomedical Engineering, University of Florida, Gainesville, Florida

\begin{abstract}
Deep brain stimulation (DBS) has emerged as a promising intervention for the treatment of select movement and neuropsychiatric disorders. Current DBS therapies deliver electrical stimulation continuously and are not designed to adapt to a patient's symptoms. Continuous DBS can lead to rapid battery depletion, which necessitates frequent surgery for battery replacement. Next-generation neurostimulation devices can monitor neural signals from implanted DBS leads, where stimulation can be delivered responsively, moving the field of neuromodulation away from continuous paradigms. To this end, the authors designed and chronically implemented a responsive stimulation paradigm in a patient with medically refractory Tourette syndrome. The patient underwent implantation of a responsive neurostimulator, which is capable of responsive DBS, with bilateral leads in the centromedian-parafascicular ( $\mathrm{Cm}-\mathrm{Pf})$ region of the thalamus. A spectral feature in the $5-$ to $15-\mathrm{Hz}$ band was identified as the control signal. Clinical data collected prior to and after 12 months of responsive therapy revealed improvements from baseline scores in both Modified Rush Tic Rating Scale and Yale Global Tic Severity Scale scores (64\% and 48\% improvement, respectively). The effectiveness of responsive stimulation ( $p=$ 0.16 ) was statistically identical to that of scheduled duty cycle stimulation ( $p=0.33$; 2-sided Wilcoxon unpaired rank-sum t-test). Overall, responsive stimulation resulted in a $63.3 \%$ improvement in the neurostimulator's projected mean battery life. Herein, to their knowledge, the authors present the first proof of concept for responsive stimulation in a patient with Tourette syndrome.
\end{abstract}

https://thejns.org/doi/abs/10.3171/2017.6.JNS17626

KEY WORDS Tourette syndrome; responsive deep brain stimulation; next-generation deep brain stimulation systems; functional neurosurgery

$\mathrm{D}$ EEP brain stimulation (DBS) has emerged as a promising intervention for the treatment of select movement and neuropsychiatric disorders. Current DBS therapies deliver electrical stimulation continuously and are not designed to adapt to a patient's symptoms. For disorders with paroxysmal symptoms, this can contribute to unwanted side effects. Moreover, continuous DBS can lead to rapid battery depletion, which necessitates frequent surgery for battery replacement. Next-generation neurostimulation devices can monitor neural signals from im- planted DBS leads. If pathology can be detected in real time, stimulation can be delivered responsively, moving the field of neuromodulation away from continuous paradigms. To this end, we chose Tourette syndrome (TS) as a model, because of the presence of paroxysmal symptoms, known as tics. In a previous study, a small cohort of patients received implantable devices so that we could study the neural signatures of tics in order to inform manufacturers when developing future responsive stimulation systems. ${ }^{10}$ During this initial stage of the study, stimulation ABBREVIATIONS DBS = deep brain stimulation; IPG = implantable pulse generator; MRTRS = Modified Rush Tic Rating Scale; NIH = National Institutes of Health; TS =
Tourette syndrome; YGTSS = Yale Global Tic Severity Scale.

SUBMITTED March 10, 2017. ACCEPTED June 2, 2017.

INCLUDE WHEN CITING Published online September 29, 2017; DOI: 10.3171/2017.6.JNS17626.

* Mr. Molina and Dr. Okun share first authorship. 
was optimized and delivered on scheduled duty cycles. Herein, to our knowledge, we present the first proof of concept for responsive stimulation for TS in a patient.

\section{Case Report}

\section{History and Examination}

This 27-year-old man with intractable TS provided informed consent to participate in a National Institutes of Health (NIH)-sponsored TS DBS study (clinicaltrials. gov, registration no. NCT01329198) that was designed as a trial of scheduled duty cycle DBS and also as a study to uncover the neural correlates of human tic generation. The patient was evaluated at the Center for Movement Disorders and Neurorestoration at the University of Florida by an interdisciplinary DBS screening team (neurology, neurosurgery, psychiatry, physical therapy, occupational therapy, and speech therapy). He was confirmed to have childhood-onset TS, which began at the age of 9 years, and he was concurrently diagnosed with obsessive-compulsive disorder, a common comorbidity of TS ${ }^{6}$ The patient exhibited severe motor and vocal tics, coprolalia, inappropriate gesturing, inappropriate touching, and self-injurious behavior. His most prominent tics included blinking; bending over, described as intense contractions of back, neck, and abdominal muscles; pushing the palm of either hand onto his forehead; subtle grunts; and a complex combination of the above. The patient manifested compulsions to touch hot stoves and to break cabinet handles and faucet heads. His condition was detrimental to his school studies and to his ability to maintain employment. His tics were refractory to multiple pharmacological interventions. He did not undergo psychobehavioral therapy and declined habit reversal therapy.

\section{Implantation Surgeries}

The patient underwent bilateral implantation of 2 RNS300 neurostimulator systems (NeuroPace) in a single-stage surgery in 2011. Two implantable pulse generators (IPGs) were surgically inserted and fastened to the skull (Fig. 1). MR images coupled with a deformable (patient-specific) brain atlas were used to plan the targets and trajectories of the depth electrodes. The MRI plan was fused to a CT scan, and this method was used to facilitate stereotactic targeting. Microelectrode recordings were used to assist in the placement of the DBS electrode, and these recordings monitored region-specific physiological markers. A single DL-244-3.5 depth electrode (4 contacts, $3.5-\mathrm{mm}$ spacing; NeuroPace) was implanted into each hemisphere in the centromedian-parafascicular $(\mathrm{Cm}-\mathrm{Pf})$ region of the thalamus (Fig. 1). This target region was chosen because of previous effectiveness studies., ${ }^{1,2,5,15}$ After final DBS lead implantation, intraoperative macrostimulation was used while the patient was still fully awake to determine the thresholds for stimulation-induced side effects. Postoperative high-resolution CT images were obtained approximately 1 month after lead placement, and the images were coregistered with the preoperative MRI study. This process confirmed the placement of the DBS leads into the intended target region.

During the first 4 years of chronic DBS therapy, the

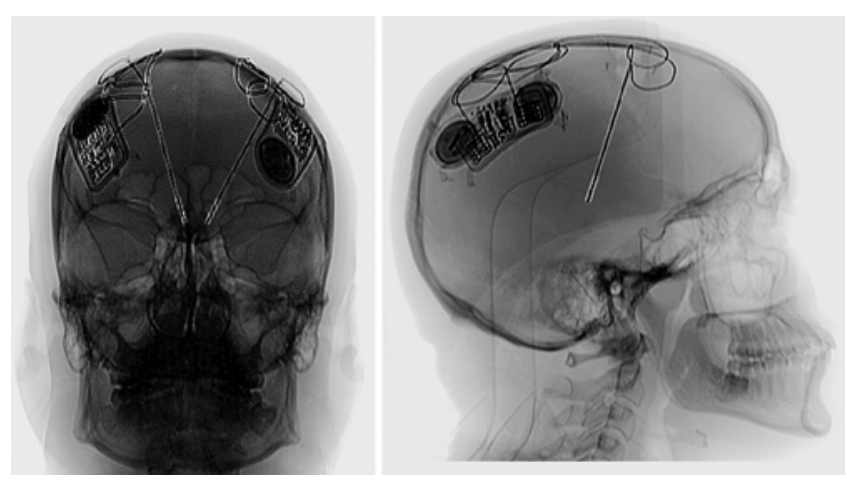

FIG. 1. Anteroposterior (left) and lateral (right) postoperative radiographs illustrating the bilateral DBS devices. Each RNS-300 IPG device is mechanically fastened to the skull, and the DBS leads are placed deep into the thalamic $\mathrm{Cm}$-Pf region.

patient required 2 IPG replacement surgeries because of battery depletion. Following an amendment to the FDA investigational device exemption, the patient underwent implantation with a responsive RNS-300M (NeuroPace) neurostimulator. At the time of the implantation, it was estimated that the previous IPG had reached complete battery depletion over the prior 6 months.

\section{Device Programming and Modeling}

The RNS-300 system was originally designed for the treatment of epilepsy. The device has been used for the detection of the electrophysiological features of seizure, and the updated RNS-300M system has undergone clinical trials for the treatment of epilepsy and has been recently approved by the FDA as a responsive neurostimulation device. ${ }^{11}$ Our research group used the RNS-300 system to study the electrophysiological underpinnings of tic generation and to develop a responsive stimulation system for tic suppression through FDA investigational device exemption approval. These neurostimulators were programmed on a scheduled duty cycle paradigm as part of the initial NIH trial, with the hypothesis that patients with TS would not need continuous stimulation for symptom relief due to the paroxysmal nature of the symptoms (Fig. 2A; third row). ${ }^{12}$ As part of this protocol, stimulation was provided during times of the day when the patient reported his most active tic manifestations (during the morning and during the workday). The RNS-300 was initially programmed to deliver 16-second bursts at 2-minute intervals. This paradigm was used for 8 preselected hours each day. Each burst consisted of a monophasic pulse train at $125 \mathrm{~Hz}$ with a pulse width of $120 \mu \mathrm{sec}$ and an amplitude of $3.5 \mathrm{~mA}$. In total, the patient received 208 bursts per day on a scheduled duty cycle therapy.

Two RNS-300M neurostimulators were implanted bilaterally during the most recent battery replacement surgery. The RNS-300M system does not support a scheduled duty cycle, and we used the information gained from our previous study to carry out a responsive stimulation paradigm..$^{10}$ The responsive stimulation mode on the RNS$300 \mathrm{M}$ can be set so that a daily limit is placed on the number of bursts. Placing these limits has the advantage of maintaining a minimum dosage, avoiding overstimula- 
$\mathbf{A}$

\section{Stimulation Paradigm}
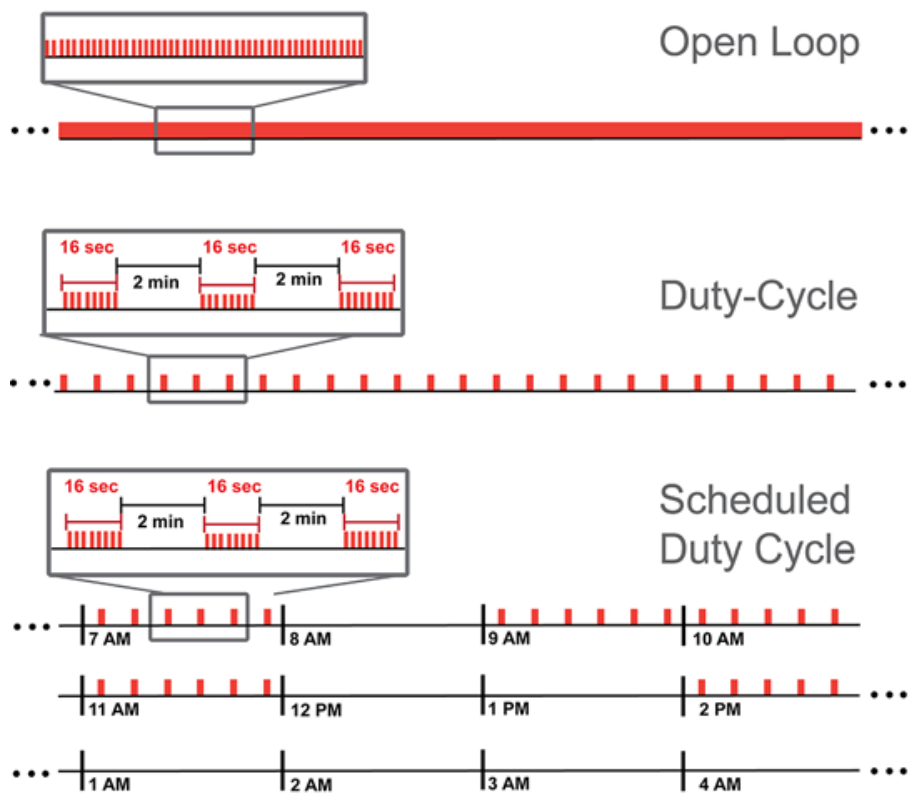

Responsive with Detection

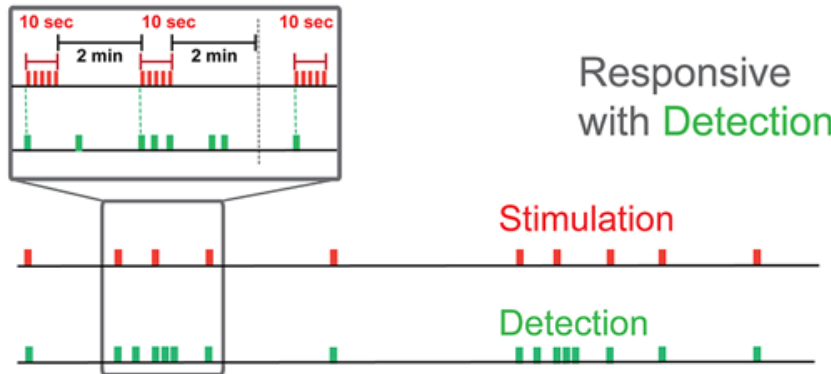

\section{$\mathbf{B}$}

\section{Daily Dosage Battery Estimates}

Open Loop
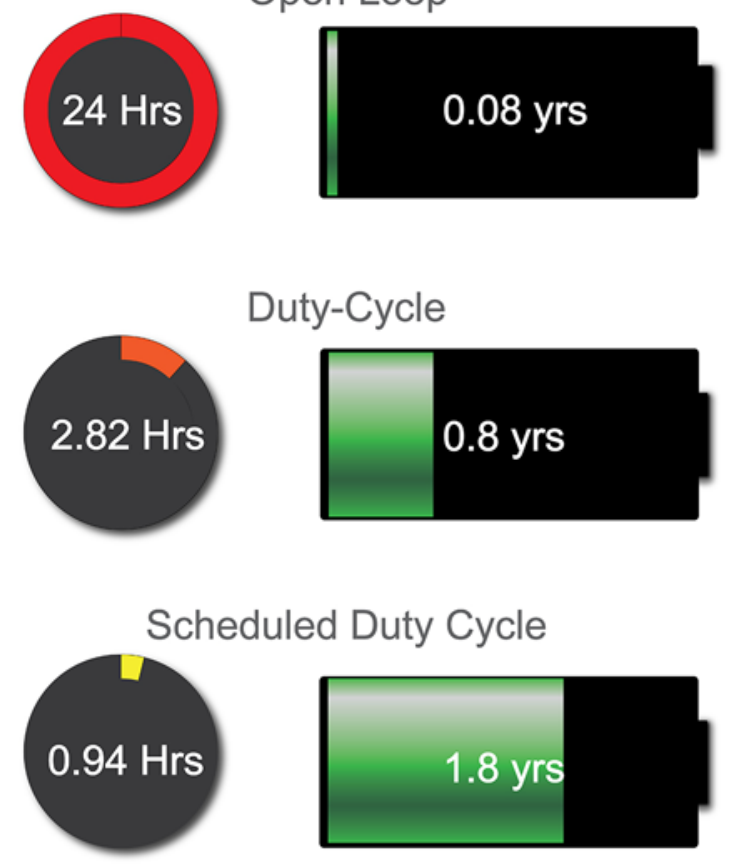

Responsive with Detection
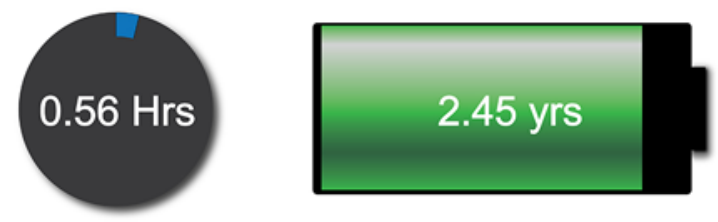

FIG. 2. Stimulation paradigms and neurostimulator battery life. A: Stimulation patterns (shown in red) delivered in 4 different paradigms. The responsive paradigm demonstrates an example of 10-second stimulation bursts upon tic detection, followed by an uninterrupted 2-minute poststimulation interval, regardless of any subsequent detections. B: Total daily dosage and the corresponding device service life. The daily dosage refers to the cumulative on time of the device, with the open-loop paradigm corresponding to 24 hours. A duty cycle of 16 seconds on/2 minutes off yields a daily dosage of 2.82 hours. Delivering the same duty cycle on schedule decreases the dosage to approximately 1 hour, and responsive stimulation can further decrease it to approximately 0.5 hour. The estimated battery life can be extended to 2.45 years on responsive stimulation. Figure is available in color online only.

tion, and preventing accelerated depletion of the device. The RNS-300M was programmed to deliver the same intensity and waveform as the previous therapy; however, it was only capable of delivering 10 -second bursts instead of the previous 16 -second bursts. The daily therapy limit was set to 200 bursts of 10 -second durations. Again, the device was set to a 2-minute refractory interval after delivery of stimulation. These settings were chosen in an attempt to control for any effects specific to stimulation parameters and to specifically compare responsive stimulation with scheduled stimulation. Stimulation was delivered by turning the device's stimulation engine on or off, without any ramping. The patient reported no adverse effects to the toggling of the stimulation.

Tic detection was performed in 4-minute trials, dur- ing which the patient was instructed to not suppress his tics. These trials were compared against trials of rest, tic imitation, and basic volitional movement (hand gestures and talking). No stimulation was delivered during these trials, and a 20 -minute washout period was allotted prior to these trials to capture as many tics as possible. These trials were videotaped, during which tics were labeled by a clinician. Initially, the detection was set to broadband low-frequency activity $(<20 \mathrm{~Hz})$, which was previously observed in this patient to correlate to higher tic severity. ${ }^{10}$ Detector parameters were further optimized until the spectral power increase in the $5-$ to $15-\mathrm{Hz}$ band yielded the best sensitivity for tics. The feature band was found by narrowing the spectral band, making sure to maintain equivalent detections during tic trials across parameters, 
while minimizing detections during rest and volitional trials. Detector optimization across both devices revealed that only the device implanted on the left yielded neural markers to discriminate complex tics (tics that involve more than 1 muscle group and last longer than 1 second) from other trial conditions. The 2 most distal electrode contacts implanted in the left side revealed the greatest sensitivity for detection when set in a bipolar configuration. Bandpass detection, using a half-wave method, was performed in 128 -msec windows, with a minimum machine-unit amplitude of 8 and a 28 machine-unit hysteresis. Parameter optimization was done in the "Tech Mode" of the clinical programmer. The amplitude threshold was chosen to maximize detection in the spectral band, which revealed tics, whereas the hysteresis threshold is meant to minimize spurious detection caused by low-magnitude changes. The device on the right side was programmed to detect the stimulation frequency $(125 \mathrm{~Hz})$ of the other device to deliver stimulation in response to the detected tics.

This optimization process of the tic detector was conducted over three 2-day visits. The number of event detections within each trial was compared with the number of tics (for true positives and false negatives) and the number of volitional movements (for false positives) to fine tune the detector parameters. Each tuning session was followed by a 30- to 45-minute trial of responsive stimulation to ensure that no adverse events occurred. The patient was discharged on a responsive stimulation setting following the 1st day of optimization. The overnight tic detection and stimulation counts collected informed the final tuning session, which was performed on Day 2. The device was also programmed to collect monthly tic detection and stimulation delivery counts. All battery life estimates were provided by the manufacturer (NeuroPace).

\section{Clinical Measures}

Acute therapeutic effectiveness of the stimulation paradigms was measured with the Modified Rush Tic Rating Scale (MRTRS), ${ }^{3}$ a video-based assessment tool scored by a clinical rater, and chronic effectiveness was measured with the Yale Global Tic Severity Scale (YGTSS). ${ }^{7}$ The scores were tallied at baseline before the neurostimulator battery change (changed to the responsive system). Scores for the previous scheduled therapy were collected over monthly visits for 6 months and during 3 semiannual visits. Scores were recorded prior to reimplantation during the period when the batteries had been completely depleted. Responsive therapy scores were collected over the course of a year (following the first programming and during 2 semiannual visits).

\section{Results}

\section{Clinical Outcome Scores}

The patient reported subjective meaningful improvement in his tics, which was supported by the clinical data collected prior to and following 12 months of responsive therapy. The patient's scores prior to battery replacement surgery, when the previous batteries were completely depleted, revealed a return to baseline tic functioning (i.e., his scores prior to any DBS therapy). Both the MRTRS

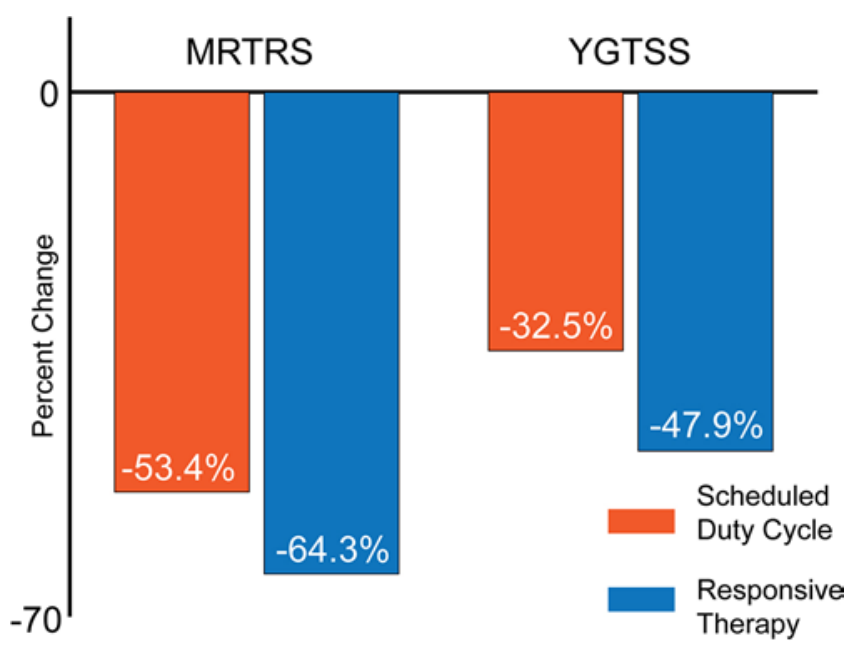

FIG. 3. Clinical score improvement. Average percent change in MRTRS (acute) and YGTSS (chronic) scores assessed on 2 different DBS paradigms. Both scores revealed a decrease in the tic severity scores, which reflect improvement (i.e., reduction) of tics. Responsive stimulation resulted in a larger decrease (improvement) on both tic scales, although we cannot state statistical difference in this study with 1 patient. Figure is available in color online only.

and the YGTSS scores revealed improvement from his baseline scores with scheduled stimulation ${ }^{12}$ and responsive stimulation (Fig. 3). The scheduled and responsive modes of stimulation resulted in a 53\% and 64\% improvement, respectively, in his MRTRS scores when compared with the pre-DBS implantation condition $(\mathrm{p}=0.0018$ and $\mathrm{p}=0.0034$, respectively; 2 -sided Wilcoxon unpaired ranksum test). The YGTSS scores, which reflect the chronic clinical outcome, showed an improvement of $33 \%$ on scheduled and a $48 \%$ improvement on responsive therapy. The effectiveness of responsive stimulation $(p=0.16)$ was statistically identical to scheduled duty cycle stimulation ( $\mathrm{p}=0.33$; 2 -sided Wilcoxon unpaired rank sum $\mathrm{t}$-test). Figure 3 provides a summary of the clinical scores and a summary of statistical comparisons.

\section{Detector Performance}

Figure 4 presents spectrograms of thalamic data when the tics were suppressed (upper) and when they were not suppressed (lower), which involved frequent long complex dystonic tics. Thalamic activity contralateral to the arm involved in tics (left thalamus) in the 5- to $15-\mathrm{Hz}$ range was found to be significantly higher during tics $(\mathrm{p}<0.01$, 2 -sided Wilcoxon unpaired rank-sum test) and was used to program the detector. The detector exhausted the preset daily therapy limit, but extended the hours of therapy from 8 hours on the scheduled paradigm to an average of 10.2 hours while on responsive stimulation. Figure 5 presents histograms of the number of detected events averaged across 12 months. Figure 5A compares the average number of detection events per day while the stimulator was active (while the stimulation dose was being delivered) versus when the stimulator was inactive (after the daily stimulation dosage was reached). The number of tics per day was significantly lower during therapy delivery than during the inactive period $(\mathrm{p}<0.001,2$-sided unpaired $\mathrm{t}-$ 

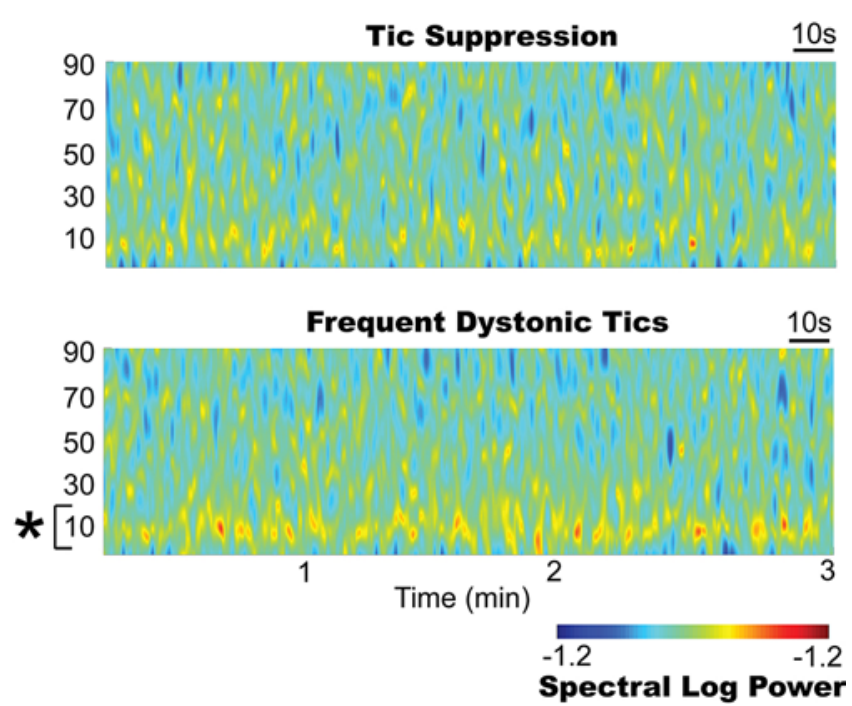

FIG. 4. Spectral features of tics. Upper: Time-frequency spectrogram of thalamic signals during a tic-free 3-minute recording. Lower: Spectrogram obtained during long dystonic tics showing the spectral power increase $\left({ }^{*} p<0.05\right)$ in the $5-$ to $15-\mathrm{Hz}$ band. Figure is available in color online only.

test). Figure 5B summarizes the distribution of the number of detected events during therapy delivery, and it details particularly stressful hours of the day which were reported to worsen tics (e.g., work hours). Two-sided unpaired Wilcoxon unpaired rank-sum tests revealed detection differences between work hours and off hours within a day, as well as work hours and rest days $(\mathrm{p}=0.045$ and 0.0019 , respectively).

\section{Neurostimulator Battery/Projections and Dosage}

Responsive stimulation resulted in a $63.3 \%$ improvement in the neurostimulator's projected mean battery life when compared with scheduled stimulation. In addition, there was a $145 \%$ improvement when compared with duty cycle-only therapy (Fig. 2B). The cumulative stimulation dosage was also calculated and resulted in a $40 \%$ and $80 \%$ reduction in the duty cycle and in the scheduled duty cycle schemes, respectively.

\section{Discussion}

This report demonstrates for the first time that longterm responsive DBS therapy in TS could be safe and feasible, with the potential to provide effective therapy for select patients. Using the NeuroPace RNS system, which has been shown to provide treatment options for intractable epilepsy, ${ }^{11}$ we designed a tic detector based on human tic-related electrophysiology, and we customized the solution to the patient's symptoms. We identified a band (5-15 $\mathrm{Hz})$ that accounted for the patient's tics and that provided a control signal for responsive stimulation. There was a significant and clinically realized benefit with chronic responsive therapy over the course of 12 months compared with baseline (53\% [MRTRS] and 33\% [YGTSS]). Although the patient felt that responsive stimulation was superior in effectiveness to the previous scheduled stimulation, the primary aim of this pilot study was not to compare the 2 approaches, but to provide a proof of concept for responsive stimulation for TS. Similarly, although responsive stimulation was safe and well tolerated in this individual, a larger study will need to address any potential benefits or worsening in the side-effect profile (i.e.,
A Suppression of Targeted Activity by DBS

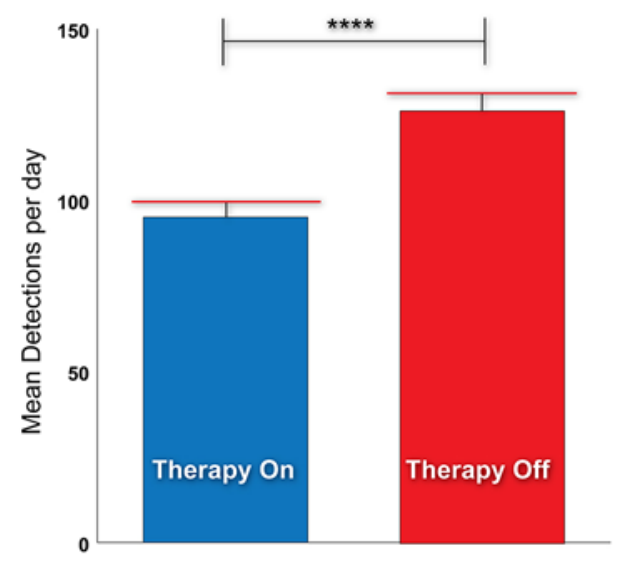

B

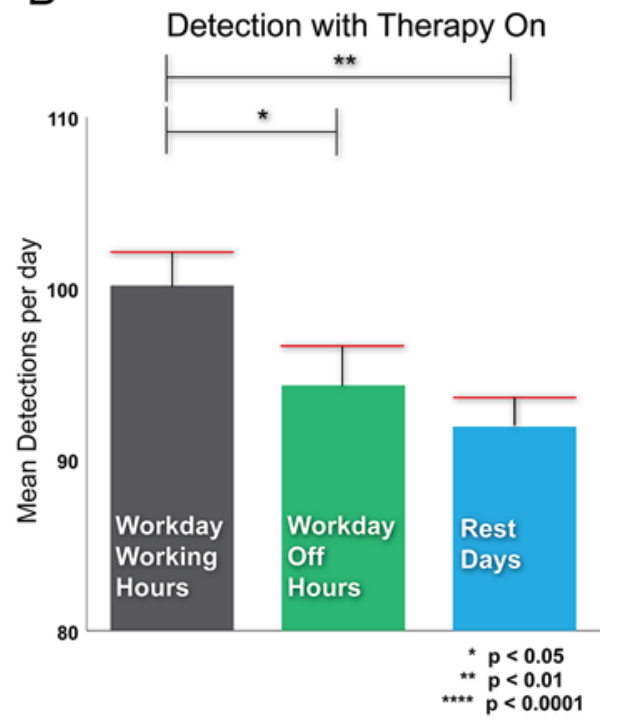

FIG. 5. Therapeutic effects of responsive DBS. A: The average number of tics detected during active responsive DBS (therapy on) was significantly lower than the number of detections after the daily therapy limit was reached (therapy off). This suggests that responsive delivery of DBS suppresses tics. B: There was also a statistically significant difference between the number of detections during the workday when comparing working hours and off hours. Similarly, there was a significant difference between working hours and rest days. These results are consistent with the patient's self-report of having stress-related tics at work. Figure is available in color online only. 
future patients may sense when the stimulation discharges and this could prove to be an intolerable scenario). Several groups are working toward this goal for other movement disorders; $4,8,9,13$ however, these studies are in their early phases and are mainly restricted to the laboratory environment.

The significant improvement in clinical outcome scores supports the potential benefits of responsive DBS therapy. The inclusion of a battery depletion time point bolsters the argument for a clinical benefit. Responsive DBS provided similar therapeutic benefits as scheduled duty cycle stimulation. There was, however, a shorter cumulative discharge time (i.e., daily dosage) in the responsive mode. These results challenge the idea of a direct correlation between total stimulation duration and clinical effectiveness; ${ }^{14}$ as in this case, less stimulation provided at least equivalent and possibly better clinical outcome. This finding should further motivate the neuromodulation research and clinical community to study the neurophysiological correlates in other neurological disorders, which may be candidates for this approach. The findings from this report of 1 patient suggest that responsive DBS therapy may possibly be tailored to the current clinical condition of an individual patient.

Distribution of detected events on and off responsive therapy revealed that DBS suppressed the 5- to $15-\mathrm{Hz}$ target band. More detections late in the day revealed that the activity band was at least partially suppressed before reaching the daily therapy limit of device discharges (Fig. 5A). Another interesting observation was that the detections correlated with the patient's unusual work schedule and his subjective description of when the tics worsened during the day (Fig. 5B). Increased stress levels have been shown to worsen symptoms of basal ganglia diseases, including TS. ${ }^{6}$ The clinical outcome scores and subjective descriptions also tracked with the detector. The total amount of stimulation (daily dosage) with the responsive paradigm, even with false-positive stimulations, is still less than the dosage with continuous and scheduled therapy. Still, finding an optimal balance will be important since the development of closed-loop technologies depends heavily on adequate modulation of pathologic activity. Moreover, the variability in tics and the changes in tics across time make the task of responsive stimulation more challenging.

The improvement of responsive systems for TS will be the next step in therapy development. As discrimination of abnormal patterns improve, cumulative stimulation times will decrease and neurostimulator battery life will be prolonged (Fig. 2). The improvement in battery life can have a significant impact. For example, an increase in device longevity that is gained from switching to responsive stimulation (from scheduled) over the course of 30 years would translate to a $25 \%$ reduction in device replacement surgeries. Since TS usually occurs in early life, and the mean age of DBS therapy is usually younger than 40 years, this could have a significant impact on cost and long-term adverse events from repeat surgeries. Finally, even if a rechargeable device becomes standard, responsive stimulation will still boost device longevity.

There are a few limitations to our study that must be acknowledged. First, only one of the devices provided neu- rosignatures that reliably correlated with tics. This finding could have been due to the lateralized nature of most of the patient's motor tics. We were, however, able to program the other device to synchronize firing with the contralateral detector. This leads to the question of whether therapy in select patients could be delivered unilaterally with similar clinical benefit. At this stage of the therapy, the patient chose to receive bilateral stimulation. Currently, the patient travels hundreds of miles away to our center and can only undergo biannual follow-up. This led to the use of a detector scheme with high sensitivity at the expense of low precision. We chose an on-demand paradigm that minimized missing tic-related pathology, even if it meant that there were several false-positive stimulations in a day. More frequent visits and adjustments to the detector model could lead to better performance in both measures. Also, we only implemented our responsive paradigm in a single patient. More patients will be needed to assess whether tic-related pathology is consistent, and whether responsive stimulation can result in symptom relief across larger populations. Finally, this study focuses on only one of all the available DBS systems. DBS devices can vary in battery size, discharge efficiency, and the load from real-time monitoring. Nevertheless, stimulation itself remains the most costly operation, and its reduction should translate to positive gains in battery longevity on other platforms.

\section{Conclusions}

DBS has emerged as a promising intervention for the treatment of select cases of TS. Next-generation neurostimulation devices have the potential to inform when stimulation should be provided, and can facilitate a responsive stimulation solution. There may be a long-term economic benefit to responsive TS DBS. Future studies will be required to address whether this approach can reduce unintended side effects, such as speech problems, walking difficulties, and cognitive dysfunction. This single case provides a proof of concept for larger follow-up studies.

\section{References}

1. Ackermans L, Duits A, van der Linden C, Tijssen M, Schruers K, Temel Y, et al: Double-blind clinical trial of thalamic stimulation in patients with Tourette syndrome. Brain 134:832-844, 2011

2. Ackermans L, Temel Y, Cath D, van der Linden C, Bruggeman R, Kleijer M, et al: Deep brain stimulation in Tourette's syndrome: two targets? Mov Disord 21:709-713, 2006

3. Goetz CG, Pappert EJ, Louis ED, Raman R, Leurgans S: Advantages of a modified scoring method for the Rush videobased tic rating scale. Mov Disord 14:502-506, 1999

4. Herron JA, Thompson MC, Brown T, Chizeck HJ, Ojemann JG, Ko AL: Chronic electrocorticography for sensing movement intention and closed-loop deep brain stimulation with wearable sensors in an essential tremor patient. J Neurosurg 127:580-587, 2017

5. Houeto JL, Karachi C, Mallet L, Pillon B, Yelnik J, Mesnage $\mathrm{V}$, et al: Tourette's syndrome and deep brain stimulation. $\mathbf{J}$ Neurol Neurosurg Psychiatry 76:992-995, 2005

6. Jankovic J: Tourette's syndrome. N Engl J Med 345:11841192,2001

7. Leckman JF, Riddle MA, Hardin MT, Ort SI, Swartz KL, Stevenson J, et al: The Yale Global Tic Severity Scale: initial 
testing of a clinician-rated scale of tic severity. J Am Acad Child Adolesc Psychiatry 28:566-573, 1989

8. Little S, Pogosyan A, Neal S, Zavala B, Zrinzo L, Hariz M, et al: Adaptive deep brain stimulation in advanced Parkinson disease. Ann Neurol 74:449-457, 2013

9. Malekmohammadi M, Herron J, Velisar A, Blumenfeld Z, Trager MH, Chizeck HJ, et al: Kinematic adaptive deep brain stimulation for resting tremor in Parkinson's disease. Mov Disord 31:426-428, 2016

10. Maling N, Hashemiyoon R, Foote KD, Okun MS, Sanchez JC: Increased thalamic gamma band activity correlates with symptom relief following deep brain stimulation in humans with Tourette's syndrome. PLoS One 7:e44215, 2012

11. Morrell MJ: Responsive cortical stimulation for the treatment of medically intractable partial epilepsy. Neurology 77:1295-1304, 2011

12. Okun MS, Foote KD, Wu SS, Ward HE, Bowers D, Rodriguez RL, et al: A trial of scheduled deep brain stimulation for Tourette syndrome: moving away from continuous deep brain stimulation paradigms. JAMA Neurol 70:85-94, 2013

13. Rosa M, Arlotti M, Ardolino G, Cogiamanian F, Marceglia S, Di Fonzo A, et al: Adaptive deep brain stimulation in a freely moving Parkinsonian patient. Mov Disord 30:1003-1005, 2015

14. Rossi PJ, Opri E, Shute JB, Molina R, Bowers D, Ward H, et al: Scheduled, intermittent stimulation of the thalamus reduces tics in Tourette syndrome. Parkinsonism Relat Disord 29:35-41, 2016
15. Servello D, Porta M, Sassi M, Brambilla A, Robertson MM: Deep brain stimulation in 18 patients with severe Gilles de la Tourette syndrome refractory to treatment: the surgery and stimulation. J Neurol Neurosurg Psychiatry 79:136-142, 2008

\section{Disclosures}

Dr. Foote states that he is a consultant for and has received research support for this study from Medtronic.

\section{Author Contributions}

Conception and design: Gunduz, Okun. Acquisition of data: Molina, Shute, Opri, Rossi. Analysis and interpretation of data: Molina. Drafting the article: Molina. Critically revising the article: Gunduz, Martinez-Ramirez. Reviewed submitted version of manuscript: Gunduz, Molina, Okun, Shute, Opri, Rossi, MartinezRamirez, Foote. Approved the final version of the manuscript on behalf of all authors: Gunduz. Statistical analysis: Molina. Administrative/technical/material support: Okun, Foote. Study supervision: Gunduz, Okun.

\section{Correspondence}

Aysegul Gunduz, University of Florida Department of Electrical and Computer Engineering, 1275 Center Dr., BMS Bldg. J283, Gainesville,FL 32611-6131. email: agunduz@ufl.edu. 